Review Article, PET/CT.

\title{
Prostate Cancer-Specific Positron Emission Tomography with Different Radiotracers.
}

\author{
Alshemeili, M. \\ Nuclear Medicine Department, Faculty of Medicine, Claude Bernard University Lyon, \\ France.
}

\section{INTRODUCTION:}

Imaging evaluation of prostate cancer needs to be tailored for various radiopharmaceuticals being remarkably heterogeneous disease. Positron emission tomography (PET) has played a relatively limited role in prostate cancer imaging. However, in recent years, several new PET tracers have emerged, offering potential improvements in diagnostic performance for both the detection of prostate cancer metastases at initial staging and the localization of recurrent disease. We reviewed the literature for prostate cancer PET tracers that are either being used for patient management or being evaluated in clinical research trials. For each tracer, we compiled clinically relevant background information and evidence supporting clinical use, with the intention of providing a high-yield primer for urologists managing patients with prostate cancer. ${ }^{18} \mathrm{~F}-\mathrm{FDG}, \quad{ }^{18 \mathrm{~F}-\mathrm{NaF},}{ }^{11} \mathrm{C}$-choline, and 18F-fluciclovine have all proven useful for prostate cancer imaging, though the utility of each of these tracers is limited to targeted management questions and particular clinical settings. In contrast, the newer prostate-specific membrane antigen (PSMA) agents may prove useful as general purpose PET tracers for prostate cancer imaging. Numerous other novel PET tracers have shown promising results in pre-clinical studies.

OBJECTIVE: Recent advances of current state of PET radiotracers for potential use in the imaging evaluation of prostate cancer.

Key Words: Prostate cancer, radiotracers, positron emission tomography.

Corresponding Author: Alshemeili, M. E-mail: malhasouni@yahoo.com. 
Prostate cancer is the most common cancer in men, with an annual incidence of 180 000 cases in the U.S.A. around 30000 deaths per year can be attributed to it ${ }^{(1)}$. Prostate cancer is locally invasive and can spread to the regional and distant lymph nodes. However, the most common cause of death is metastasis to the bone and bone marrow, which results in progressive debility and hematopoietic involvement. Prostate cancer is a disease of the elderly, with patients with this condition having an average age of 63 years. Incidence increase progressively with age. However, the majority of patients with prostate cancer have no disease symptoms during their life time. About one-third of men over 50 years of age have prostate cancer, discovered incidentally during autopsy. (Ref .Nuclear medicine in clinical diagnosis et treatment (2). PET is fundamentally suited for the imaging evaluation of biologic targets and events. With the ever-increasing availability and access to hybrid PET/CT scanners, as well as the recent emergence of PET/MRI systems coupled with exceptional ongoing research and development in radiochemistry, the future for noninvasive molecular imaging-based examination of prostate cancer biology not only will lead to new enlightening levels of understanding of the disease but also will be clinically useful. Prostate cancer recurrence, following primary treatment, is usually indicated by rising serum prostatespecific antigen (PSA) levels, which can provoke anxiety in patients and clinicians. Fortunately, advances in pcPET have facilitated newer insights into the patterns of disease recurrence. Emerging pcPET radiotracers including $\mathrm{C}-11$ choline, Ga-68 - PSMA, C-11 acetate, and FACBC have the ability to localize prostate cancer recurrence at an earlier stage in the disease course, when PSA levels are low, to inform medical decision-making, and to investigate PET-directed local therapy. Although there have been several narrative review articles on this evolving topic, formalized systematic reviews and metaanalyses for a few radiotracers, have only recently appeared in the literature (Table 1). 
Table 1: Positron emission tomography in prostate cancer.

\begin{tabular}{|c|c|}
\hline Radiotracer & Process \\
\hline${ }^{18}$ F-FDG & Glycolysis \\
${ }^{18}$ F-fluoride & Hydroxyapatite crystal \\
${ }^{11}$ C-acetate & Fatty acid synthesis \\
${ }^{11}$ C-methionine & Amino acid transport \\
${ }^{11}$ C-choline & Phospholipid synthesis \\
${ }^{18}$ F-fluoroethylcholine & Phospholipid synthesis \\
${ }^{18}$ F-methylcholine & Phospholipid synthesis \\
${ }^{18}$ F-cis-4-L-proline & Amino acid transport \\
${ }^{18}$ F-fluoromisonidazole & Hypoxia \\
\hline
\end{tabular}

\section{Fluoro-2-Deoxyglucose:}

It is a radiopharmaceutical product most commonly used for PET. Fluorodeoxyglucose (FDG) is glucoseanalog, which has been rendered radioactive. Malignant tumor cells consume a considerable amount of sugar, because of their high energy requirement. This molecule is effective in the detection of several malignancies, including lymphoma, breast cancer, lung cancer, and Head and Neck cancer.

Unfortunately, FDG is not effective for prostate cancer detection, owing to the slow progress of prostate cancer. Thus, have low energy consumption. However, aggressive forms of prostate cancer have high $\mathrm{FDG}^{(3)}$.
[18F] sodium fluoride and detection bone metastases:

Bone metastasis is frequently associated with progressive prostate cancer. Bone scintigraphy is still the standard examination reference for detecting these metastases. To improve the image quality of PET and whole body tomographic examination coupled to $\mathrm{CT}$ in a reasonable image acquisition time, a plotter accumulating in the forming bone is available since several years: $[18 \mathrm{~F}]$ sodium fluoride (FNa). Its performance was compared with that of FDG and its performance appeared to be superior, regardless of the aggressiveness of the disease [castration-resistant prostate cancer(CPRC), high Gleason score], testifying once again the more limited role of FDG in prostate cancer detection and of the need for new tracers ${ }^{(4)}$. 
Lipid PET tracers: choline (11C) and fluorocholine (18F):

A lipid likes choline if rendered radioactive; it can help detect those sites, which are associated with higher synthesis of cell membranes, than that of normal tissue. Prostate cancer exhibits increased metabolism of phosphatidylcholine, which is an essential component of the cell membrane, by increasing the rate of choline kinase and the rate of incorporation of choline. The radioactive derivatives of choline labeled11C and then $18 \mathrm{~F}$, mainly $118 \mathrm{~F}-\mathrm{FCH}$, were developed several years ago and studied first in animals and subsequently in humans. The efficacy of $18 \mathrm{FCH} \mathrm{PET} / \mathrm{CT}$ is controversial for the initial assessment of the extent of prostate cancer and / or primary tumor detection in The dose equivalent effective amount is $0.03 \mathrm{mSv} / \mathrm{MBq}$. The organ that receives the highest dose is the kidney $(0.17 \mathrm{mSv} /$ $\mathrm{MBq})$. Choline belongs to the family of amino alcohols. It is found in the diet or is endogenously synthesized from glycine in the liver. In order to limit the exogenous intake of choline and a possible phenomenon of intracellular trapping, the patient is advised to fast for four hours before the examination.

A dynamic acquisition of the diagnostic image lasting ten minutes is started immediately after intravenous injection patients with negative biopsy results and high PSA levels.

Several analogs of choline have been studied for the detection of prostate cancer and brain tumors. The most frequently used analogs are18F-FCH and fluoromethyl-(18F)-dimethyl-2-

hydroxyethyl-ammonium. The absorption rate and the phosphorylation rate of $18 \mathrm{~F}$ $\mathrm{FCH}$ in tumor cell lines, in vitro, were significantly higher than those observed for $18 \mathrm{~F}-\mathrm{FEC}$ and $18 \mathrm{~F}$-fluoro-propylcholine (FPC), making it the best tracer of choline metabolism. 18-FCH metabolism is identical to that of choline: it can cross the cell membrane with the help of a specific transporter and is synthesized in to phosphatidylcholine, after phosphorylation by choline kinase ${ }^{(5)}$.

ofto $220 \mathrm{MBq}$ of 18F-FCH. This acquisition dynamic can detect a possible accumulation in the prostate gland and / or the loco regional ganglia, in the first two minutes after injection, before urinary excretion of $18 \mathrm{FCH}$. The reconstruction of these ten first minutes, in two consecutive sequences, is useful to differentiate a possible nonspecific, inflammatory accumulation of capture tumor. Once the first acquisition is complete, the patient is hydrated orally and intravenously, and is administered20 $\mathrm{mg}$ furosemide to accelerate urinary excretion. 
A trunk acquisition is usually obtained 60-75 minutes after injection, to assess the extent of the tumor.

\section{FCH localization of prostate cancer:}

Several studies have evaluated the efficacy of PET with FCH in the detection of prostate cancer in the prostatic lodge, with discordant results. It was initially concluded that FCH could not reliably differentiate between prostate cancer and benign hyperplasia ${ }^{(6)}$. Recent data has shown that the area of the prostate invaded by cancer showed significantly higher absorption of $\mathrm{FCH}$, than the areas without the tumor (Figure 1).

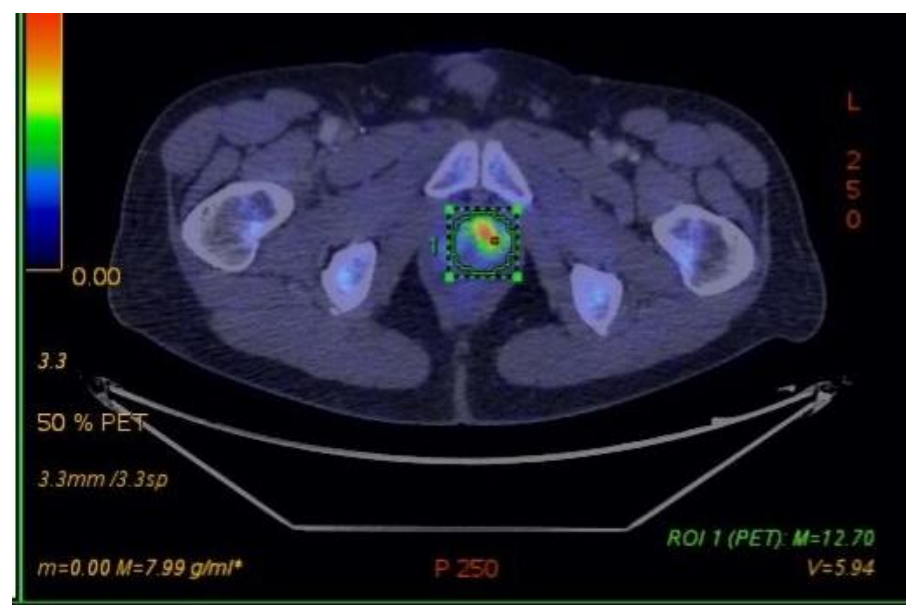

Figure 1: ${ }^{18} \mathrm{~F}$ choline PET-CT. Fused axial section of pelvis, showed intense hyper metabolism focus in left prostatic lodge.

On the other hand, the acquisition of images one hour after injection is needed for the differentiate between benign hyperplasia and malignant prostate cancer. It would even allow for guided biopsies in difficult cases, in patients with repeatedly negative initial samples ${ }^{(4)}$. PET /magnetic resonance imaging (MRI) with $\mathrm{FCH}$ is a newer approach compared to conventional MRI of the detection of prostate cancer ${ }^{(7)}$. FCH PET / CT were also used to determine the target biological tumor volume for prostate cancer radiotherapy.
Pinkawa et al,. devised a FCH PET / CT for radiotherapy planning in 66 patients with prostate cancer. Two years later, the same team prospectively assessed the quality of life of patients after radiotherapy, with $(n=46)$ or without $(n=$ 21), radiotherapy boost, based on $\mathrm{FCH}$ PET / CT. It was concluded that the planning of PET/CT radiotherapy allows an increase in the dose delivered to the intra prostatic lesion, macroscopically, without significantly worsening the quality of life ${ }^{(8)}$. 
Iliac lymph nodes, may be involved in the early stage of the tumor can be resected quite easily) extends a little beyond the prostatic tissue. This also has prognostic value. If the lymph nodes are involved on choline-aided PET, it is indicative of their involvement in the tumor. On the other hand, the ganglia can be affected in a very subtle manner, which cannot be detected with MRI, or PET choline. If the cholinePET scan is positive, we can be certain of lymph node involvement.

It is possible to have non-regional lymph nodes involvement affected lymph nodes located higher, especially in the aortic region.

Amino Acid Metabolism with Anti-18F FACBC (F-18 fluciclovine):

Anti-18F-FACBC is a synthetic non metabolized analog of the amino acid leucine that accumulates in prostate cancer cells through the up regulated alanine, serine, cysteine (ASC) transport system ${ }^{(9)}$. It has been observed recently that androgens augment the expression of the ASC transporter system, thereby, increasing the uptake of anti-18F-FACBC in prostate cancer ${ }^{(10)}$. Ren et al,. reported meta-analysis of 6 studies comprising 251 patients in whom recurrent prostate carcinoma was suspected and who underwent anti-18F-FACBC PET/CT.
The authors reported a sensitivity and specificity of $87 \%$ and $66 \%$, respectively, on a single patient-based analysis in detecting prostate carcinoma recurrence (11)

\section{Gallium 68 PSMA:}

PSMA is highly overexpressed in prostate cancer cells as a Tran's membrane protein. PSMA is a folate hydrolase found in other malignant and benign tumors as well. Before malignant transformation, of the cell surface glycoprotein, PSMA is localized to the cytoplasm and the apical side of the prostate epithelium that lines the prostatic ducts. The function of cytoplasmic PSMA is not fully understood; however, as malignant transformation occurs, PSMA is transferred to the luminal surface of the prostatic ducts. PSMA expression has been shown to be widespread in most prostate tumors, even when PSA staining is negative or weak. Increased-PSMA expression has also been observed when the tumor becomes castration-resistant ${ }^{(\mathbf{1 2})}$.

PSMA has emerged as 1 of the most favorable targets for PET imaging. Prostate cancer PSMA overexpression has been shown to be 100 - to 1000 -fold that of normal tissue expression; furthermore, PSMA expression may increase as tumor grade and castrate resistance increases ${ }^{(\mathbf{1 3})}$. 
PSMA is hypothesized to have a transport function because it internalizes ligands similar to J591, a monoclonal antibody that targets the extracellular domain. In general, targeted antibodies have presented challenges as diagnostic radiopharmaceuticals with their long circulating half-life and resultant high nonspecific background-to-tumor noise. Consequently, the more recent focus of PSMA radiopharmaceutical development has focused on small-molecule inhibitors that target the active substrate recognition site. Eder et al first described the most commonly used PSMA inhibitor in PET imaging, Ga-68 PSMA-HBED-CC, also known as Ga-68 PSMA-11, which also is internalized and accumulates in high levels even in small metastases ${ }^{(\mathbf{1 4})}$.

There appears to be growing interest in developing an 18-F-labeled PSMA agent. Some experts argue that it would offer advantages with respect to availability, production amount, and image resolution.

Ga-68 PSMA was recently evaluated by Perera et al,. In which 16 articles including 1309 patients were evaluated when evaluating on a per-patient basis, the summary sensitivity and specificity were identical at $86 \%$. When analyzed on a perlesion basis, summary sensitivity was $80 \%$ and specificity was 97\%. Additionally, it was noted that patients with biochemical recurrence had increasingly positive Ga-68
PSMA PET scans as the pre-PET PSA increased. They found that $58 \%$ were positive at a pre-PET PSA of 0.2 to 1 $\mathrm{ng} / \mathrm{mL}$, which increased to $76 \%$ with a PSA of 1 to $2 \mathrm{ng} / \mathrm{mL}$ and further increased to $95 \%$ for PSA $>2 \mathrm{ng} / \mathrm{mL}^{(15)}$.

Recently, PET imaging using PSMAligands has gained attention as a promising new radiotracer in patients with prostate cancer. Several studies promise accurate staging of primary prostate cancer and restaging after biochemical recurrence with 68Ga- PSMA ligand PET/CT. However, prospective trials and clinical guidelines for this new technique are lacking (Figure 2).

Ga-PSMA PET/CT can detect lesions characteristic for PC with improved contrast when compared to standard [16].F-fluoromethylcholine PET/CT, especially at low PSA levels.

F-18 Fluciclovine: Prostate cancerspecific PET scans are performed uniquely. Unlike standard F-18 PET scans, which are usually imaged starting at the head and scan toward the feet, pcPET scans typically image the pelvis first. Imaging is initiated 3 to 5 minutes after radiotracer administration and scanning begins at the mid-thigh and proceeds to the base of skull. This is done primarily to minimize urinary tract contamination, but also because of the short half-life of isotopes such as C-11. 


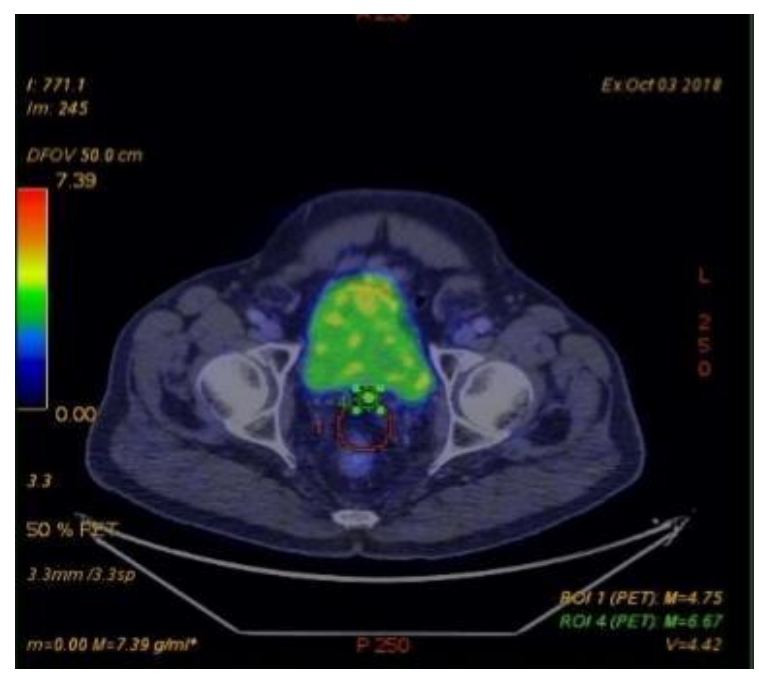

Figure 2: 68Ga-PSMA PET-CT. Fused axial section of pelvis showing hyper metabolism focus in left seminal vesicle and a nodule in contact with bladder.

Urinary tract contamination is the primary reason pcPET protocols are performed in this manner, including those involving radiotracers with longer half-lives such as F-18 fluciclovine. Fluciclovine is a synthetic amino acid, and an analog of 1leucine, which is preferentially taken up by prostate cancer cells and gliomas via specialized amino acid transporters, namely alanine-serine-cysteine transporter 2 (ASCT2) and LAT-1 ${ }^{(17)}$. Its chemical name is anti-1-amino-3-FACBC, and is commonly known by its trade name Axumin. Amino acid transporters such as ASCT2 play a critical role in amino acid metabolism in prostate cancer cells. ASCT2 is an important transporter of glutamine, which is known to be an essential tumor nutrient and has been implicated in cancer signaling pathways (18) Fluciclovine is predominantly transported by ASCT2 and transports in a manner similar to glutamine. Unlike glutamine, however, 18-F fluciclovine does not undergo additional metabolism in the cell, which lends to its intracellular accumulation particularly in prostate cancer cells and at major sites of amino acid metabolism such as the liver and pancreas ${ }^{(19)}$.

F-18 fluciclovine was evaluated by Ren et al and included 6 studies including 251 patients with biochemical recurrence ${ }^{(20)}$. The pooled sensitivity and specificity on a per-patient analysis was $87 \%$ and $66 \%$, respectively; however, caution should be exercised when interpreting the specificity in this meta-analysis. Two recent important papers evaluating the operational characteristics of F-18 fluciclovine were not included in this analysis. Schuster et al reported specificities of $40 \%$ and $97 \%$ for prostate bed and extra prostatic lesions, respectively ${ }^{(21)}$. 
Odewole et al,. Similarly demonstrated specificities of $56 \%$ and $100 \%$ for prostate bed and extra prostatic lesions, respectively ${ }^{(22)}$. These data indicate that the specificities may be higher than the meta-analysis suggests, particularly for extra prostatic disease.

\section{C-11 choline:}

Choline metabolism has been shown to be altered in prostate cancer cells. Increased levels of choline compounds concentrate preferentially in human prostate cancer cells derived from metastases (23). Alteration of choline metabolites within the cancer cell relates to choline transport, incorporation, and utilization within the cell $^{(24)}$.

C-11 choline was also recently evaluated by Fanti et al, specifically looking at its ability to detect sites of recurrence in the setting of biochemical recurrence after definitive treatment ${ }^{(25)}$. There were 12 studies including 1270 patients to derive a pooled sensitivity and specificity of $89 \%$. This was similar to previously published meta-analyses by Umbehr et al,. Fanti et $\boldsymbol{a l}$,. Highlight the accuracy of C-11 choline PET at different sites of recurrence, reporting a decreased pooled sensitivity of $61 \%$ for detection of local recurrence ${ }^{(26)}$. This result is consistent with comparative studies that have shown multipara metric
MRI with end rectal coil to be superior to C-11. choline for the detection of local recurrence, whereas C-11 choline PET/CT was shown to be superior to MRI for pelvic lymph node metastases and equal with respect to bone metastases ${ }^{(27)}$.

F-18 PSMA radiotracer was developed and is currently licensed to Cyclotek for clinical use in Australia and New Zealand (28). Since the development of F-18 DCFBC, second-generation tracers such as F-18 DCFPyL have been developed ${ }^{(34)}$. Currently, there are a number of groups working to develop the most clinically useful next-generation F-18-labeled PSMA radiotracer ${ }^{(29)}$. PSMA's unique expression differential between cancer and normal cells coupled with its large extracellular domain provides an excellent target for imaging, but also for therapeutics such as Theranostics applications with lutetium 177 PSMA. Less than 10\% of prostate cancers have no uptake on PSMA PET ${ }^{(30)}$. Additionally, the short half-life of Ga-68 (68 minutes) results in low radiation exposure to patients. Furthermore, the agent is rapidly cleared from nontarget tissue. On average, patients receive 3.0 $\mathrm{mSv}$ from the PET component of $150 \mathrm{MBq}$ of Ga-68-PSMA-11, which is lower than most other pcPET agents such as C-11 and 18-F choline scans ${ }^{(31,32)}$. 
Additional pcPET radiotracers used in prostate cancer imaging have been developed as previously noted. These include C-11 acetate and F-18 sodium fluoride. In addition, F-18 PET may be useful in imaging prostate cancer patients who have developed dedifferentiated neuroendocrine tumors of the prostate which conversely may not image well using these pcPET agents ${ }^{(33)}$.

\section{Comparative investigations of PET radiotracers:}

In recent years, numerous systematic reviews and meta-analyses have been published evaluating the pooled operational characteristics of various pcPET radiotracers in the setting of prostate cancer recurrence .These reports are often analyzed on a per-patient or perlesion basis. Caution should be exercised in interpreting sensitivities and specificities because a comparative gold standard such as histologic confirmation is not always available. The focus of this review is recurrent disease; consequently, operational characteristics are emphasized in the setting of biochemical recurrence after definitive treatment.

The use of these pcPET agents in initial staging, response to therapy, and radiation therapy planning are of great interest but beyond the scope of this review.

Important factors to consider when interpreting operational characteristics of various pcPET radiotracers include the reference standard used to establish positive and negative proof, particularly with respect to extra prostatic disease, because these sites can be challenging to obtain histologic confirmation. Furthermore, whether the analysis was performed on a per-patient or per-lesion basis provides additional insight into the interpretation of data.

For example, many studies relied on histologic confirmation per-patient for positivity of extra prostatic disease, given it would be impractical to sample every PET avid site, whereas studies that used a per-lesion analysis often used a nonhistologic method of disease confirmation, which is subject to study examination bias (34).

A prospective study by Morigi et al,. compared Ga-68 PSMA with F-18 fluoromethylcholine ${ }^{(35)}$.The findings from this study were that Ga-68 PSMA was better than F-18 fluoromethylcholine in patients with biochemical failure. It should be noted, however, that this study used low administered choline doses $(3.5 \mathrm{MBq} / \mathrm{kg}$ ) and a slightly different radioisotope, F-18 fluoromethylcholine, as opposed to C-11 choline. Additional data comparing PSMA to choline come from retrospective series. Afshar-Oromieh et al,. evaluated 37 patients with biochemical recurrence that underwent scans with both F-18 fluoromethylcholine and Ga-68 PSMA PET/CT within 30 days of 1 another ${ }^{(36)}$. 
The authors concluded that PSMA offered a higher detection rate, higher maximum standardized uptake value, and higher tumor-to-background ratio when compared with the F-18 fluoromethylcholine scan. Schwenck et al,. Retrospectively compared Ga-68 PSMA-11 with C-11 choline and demonstrated a higher detection rate with PSMA. Interestingly, however, of the 67 patients with biochemical recurrence, 458 lymph node metastases were detected. Although 39\% were exclusively identified with Ga-68 PSMA, there were $6 \%$ identified with $\mathrm{C}-11$ choline only, and the majority $(55 \%)$ were identified by both.

The advantage of PSMA, and the clinical situation in which the majority of PSMAonly detection took place, was in patients presenting with PSA levels $<1 \mathrm{ng} / \mathrm{mL}^{(37)}$.

Comparisons between F-18 FACBC and C-11 choline have largely been undertaken by Nanni et al,. Before 2016, 3 preliminary studies comparing these 2 imaging modalities in patients with biochemical recurrence were published ${ }^{(38)}$. These studies reported favorable detection rates for fluciclovine compared with choline and provided background for the publication of their prospective trial.[39] The authors showed that, in patients with biochemical relapse after prostatectomy, F18 FACBC had higher sensitivity and specificity compared with C-11 choline ( $37 \%$ and $67 \%$ vs $32 \%$ and $40 \%$ ).
They emphasized that F-18 FACBC had better true-positive findings at lower PSA levels $(<1 \mathrm{ng} / \mathrm{mL})$ with $6 / 28(21 \%)$ patients with F-18 FACBC versus 4/28 (14\%) patients with $\mathrm{C}-11$ choline. A major limitation of this trial, and a limitation of many imaging studies evaluating operational characteristics, is the use of a suboptimal reference standard. The standard of reference in this particular study was reevaluation of the clinical and imaging history after following patients for an average of 1 year. In some cases, this meant histologic confirmation including $31 \%(4 / 13)$ of patients with positive local relapse, $15 \%(4 / 26)$ of patients with positive lymph nodes, and $0 \%(0 / 7)$ of patients with positive bone lesions. For most cases, however, the standard of reference was by repeat imaging or PSA trend after therapy. Furthermore, a low choline dose $(3.4 \quad \mathrm{MBq} / \mathrm{kg})$ was administered, which may limit the study's generalizability particularly for centers that use higher choline doses.

There have been no direct comparisons between fluciclovine and Ga-68/F-18 PSMA to date. Schuster et al prospectively evaluated patients with biochemical recurrence comparing F-18 FACBC against indium 111 capromabpendetide (ProstaScint), a radiolabeled monoclonal antibody that binds to PSMA. 
This study showed FACBC performed better than ProstaScint, demonstrating FACBC's superiority in detecting more prostatic and extra prostatic disease and effectively upstaging $25 \%$ of patients. A major strength of this study was the high incidence of pathologic confirmation of true positives, with $96 \%$ (74/77) of index lesions histologically confirmed including 55 prostate bed and 22 extra prostatic lesions ${ }^{(40)}$.

\section{Clinical application of prostate cancer- specific PET imaging:}

Patients with a rising PSA after definitive therapy often ask the clinically relevant question, "Where is the origin of my rising PSA?" Before the advent of widespread clinical use of pcPET radiotracers and multipara metric MRI, clinicians relied on suboptimal tools, primarily bone scans and CT scans, to explore the answer to this question. PcPET radiotracers are able to detect sites of recurrence when the PSA level is low, even PSA levels $<1.0 \mathrm{ng} / \mathrm{mL}$. By comparison, bone scans detect osseous metastases at a median PSA level of 40 ng/mL ${ }^{(41)}$. Abuzallouf et al,. reviewed 23 studies evaluating bone scans in newly diagnosed cases of prostate cancer and showed osseous detection rates of $2.3 \%$ for PSA $<10 \mathrm{ng} / \mathrm{mL}, 5.3 \%$ for PSA 10.1 to $19.9 \mathrm{ng} / \mathrm{mL}$, and $16.4 \%$ for PSA 20.0 to
$49.9 \mathrm{ng} / \mathrm{mL}^{(42)}$.

In the same review, 25 studies evaluating CT scans found lymph node metastases in $0 \%$ of patients with PSA $<20 \mathrm{ng} / \mathrm{mL}$ and $1.1 \%$ of patients with PSA $>20 \mathrm{ng} / \mathrm{mL}$.

Additional evidence from a prospective population-based analysis of newly diagnosed prostate cancer showed CT scan detection rates were $<15 \%$ for patients with PSA levels between 4 and $20 \mathrm{ng} / \mathrm{mL}$ (43). Based on these poor positive yields, the overall use of bone scan and CT imaging has declined in pretreatment evaluation, which has also translated to limited use in the recurrent setting ${ }^{(\mathbf{4 4 , 4 5})}$. As pcPET radiotracers improve, identification of the origin of PSA relapse is occurring at lower PSA levels than ever before demonstrated. The detection rate increases to $74 \%$ when PSA rises above $1.0 \mathrm{ng} / \mathrm{mL}$, and surpasses $90 \%$ once the PSA level is $>2.0 \mathrm{ng} / \mathrm{mL}$. Similar, albeit lower, trends are observed with cholineand fluciclovine-based radiotracers. Sensitive functional imaging has led to patterns of recurrence studies that provide insight into how prostate cancer spreads early on in the process of metastasis in a variety of clinical scenarios including post prostatectomy, post-definitive radiation therapy and post prostatectomy radiation therapy. 
Patterns of recurrence studies, such as these and others, have prompted further discussion regarding additional local therapy directed to the at-risk nodal basins or aggressive metastasis-directed therapy ${ }^{(46)}$.The era of functional imaging has arrived, and clinicians around the globe are using this technology to develop customized radiation therapy plans. In a recent meta-analysis by $\boldsymbol{O s t}$ et al,. metastasis-directed therapy to regional and distant recurrences included $66 \%$ of patients receiving radiation therapy. The authors found that $51 \%$ of patients were progression free 1 to 3 years after salvage metastasis-directed therapy ${ }^{(47)}$.

Toxicity evaluation revealed metastasisdirected radiation therapy was well tolerated, with $8.5 \%$ of patients experiencing grade 2 toxicities and 1 case of grade 3 toxicity. Retrospective data coupled with growing experience using

\section{REFERENCES:}

1. Schmid DT, John H, Zweifel R, et al. Fluorocholine PET/CT in patients with prostate cancer: initial experience. Radiology. 235: 623-88; 2005.

2. Kwee SA, Wei H, Sesterhenn I, et al. Localization of primary prostate cancer with dual-phase 18F-fluorocholine PET. J. Nucl. Med. 47:262-9; 2006.
pcPET-directed therapy have prompted the development of prospective studies. In addition to pcPET-directed external beam radiation therapy, there is also growing experience regarding Theranostics applications; the most commonly discussed being lutetium 177 PSMA $^{(48)}$.

\section{CONCLUSION:}

These variable radiotracers aim to locate the intra-prostatic tumor with greater sensitivity and specificity than MRI, in order to guide biopsy and radiotherapy, and to detect ganglionic metastases with better sensitivity and specificity. $\mathrm{FCH}$ showed excellent ability in detecting CPRC and evaluating the response to the therapy. Diagnostic follow-up using Ga 68 PSMA or Fluorine 18 PSMA are better tracer in initial evaluation and follow up of prostate cancer.

\section{Igerc I, Kohlfurst S, Gallowitsch} $\boldsymbol{H J}$, et al. The value of $18 \mathrm{~F}-$ choline PET/CT in patients with elevated PSAlevel and negative prostate needle biopsy for localisation of prostate cancer. Eur. J. Nucl. Med. Mol. Imaging, 35: 976-83; 2008 
4. Pinkawa M, Piroth MD, Holy R, et

al. Dose-escalation using intensity modulated radiotherapy for prostate cancer - evaluation of quality of life with and without 18F-choline PET-CT detected simultaneous integrated boost. Radiatoncol, 7;14; 2012.

5. Oka S, Okudaira H, Yoshida Y, et al. Transport mechanism of trans-1-amino3-fluoro[1-(14)C]cyclobutanecarboxylic acid in prostate cancer cells. Nucl. Med. Biol. 39:109-119; 2012.

6. Okudaira H, Oka S, Ono M, et al. Accumulation of trans-1-amino-3[(18)F] fluorocyclobutanecarboxylic acid in prostate cancer due to androgen-induced expression of amino acid transporters. Mol. Imaging Biol. 16:756-764; 2014.

7. Evans MJ, Smith-Jones PM, Wongvipat $J$, et al. Noninvasive measurement of androgen receptor signaling with a positron-emitting radiopharmaceutical that targets prostatespecific membrane antigen. Proc. Natl. Acad. Sci. U S A. 108:9578-9582; 2011.

8. Ren J, Yuan L, Wen G, et al. The value of anti-1-amino-3-18Ffluorocyclobutane-1-carboxylic acid $\mathrm{PET} / \mathrm{CT}$ in the diagnosis of recurrent prostate carcinoma: a meta-analysis. Acta. Radiol; 2015.

9. S Surti. Update on time-of-flight PET imaging J. Nucl. Med. 56, pp. 98-105; 2015.
10. O'Doherty J, McGowan DR, Abreu C, et al .Effect of Bayesianpenalized likelihood reconstruction on [13N]-NH3 rest perfusion quantification, $\mathrm{J}$. Nucl. Cardiol. 24 (1), pp. 282-290; 2017.

11. Ackerstaff E, Pflug BR, Nelson $J B$, et al. Detection of increased choline compounds with proton nuclear magnetic resonance spectroscopy subsequent to malignant transformation of human prostatic epithelial cells ,Cancer Res. 61, pp. 3599-3603; 2001.

12. Hernandez-Alcoceba $R$, Saniger L, Campos J, et al. Choline kinase inhibitors as a novel approach for ant proliferative drug design Oncogene, 15, pp. 2289-2301; 1997.

\section{Lima AR, Bastos Mde L, Carvalho} $\boldsymbol{P}$, et al. Biomarker discovery in human prostate cancer: An update in metabolomics studies Transl. Oncol. 9, pp. 357-370; 2016.

\section{Awwad HM, Geisel J, Obeid R.} The role of choline in prostate cancer Clin. Biochem, 45, pp. 1548-1553; 2012.

15. Muller SA, Holzapfel K, Seidl C, et al. Senekowitsch-Schmidtke Characterization of choline uptake in prostate cancer cells following bicalutamide and docetaxel treatment Eur. J. Nucl. Med. Mol. Imaging, 36. pp. 14341442; 2009. 
16. Lodi $A$ and Ronen SM Magnetic resonance spectroscopy detectable metabolomics fingerprint of response to antineoplastic treatment P. LoS. One, 6, p. e 26155; 2011.

17. Leek J, Lench N, Maraj B, et al. Prostate-specific membrane antigen: Evidence for the existence of a second related human gene Br. J. Cancer, 72, pp. 583-588; 1995.

18. Maurer T, Eiber M, Schwaiger M, et al. Current use of PSMA-PET in prostate cancer management Nat. Rev. Urol. 13, pp. 226-235; 2016.

19. Birtle AJ, Freeman A, Masters JR, et al. Tumour markers for managing men who present with metastatic prostate cancer and serum prostate-specific antigen levels of $<10 \mathrm{ng} / \mathrm{mL}$ BJU Int, 96, pp. 303$307 ; 2005$.

20. Evans MJ, Smith-Jones PM, Wongvipat J, et al. Non-invasive measurement of androgen receptor signalling with a positron-emitting radiopharmaceutical that targets prostatespecific membrane antigen Proc. Natl. Acad. Sci. U S A, 108, pp. 9578-9582; 2011.

21. Silver DA, Pellicer I, Fair WR, et al .Prostate-specific membrane antigen expression in normal and malignant human tissues Clin. Cancer Res. 3, pp. 81-85; 1997.
22. Bostwick DG, Pacelli A, Blute M, et al. Prostate specific membrane antigen expression in prostatic intraepithelial neoplasia and adenocarcinoma: A study of 184 cases Cancer, 82, pp. 2256-2261; 1998.

23. Foss CA, Mease RC, Fan H, et al. Radiolabelled small-molecule ligands for prostate-specific membrane antigen: In vivo imaging in experimental models of prostate cancer Clin. Cancer Res, 11, pp. 4022-4028; 2005.

24. Cho SY, Gage KL, Mease RC, et al. Bio distribution, tumour detection, and radiation dosimetry of $18 \mathrm{~F}-\mathrm{DCFBC}$, a lowmolecular-weight inhibitor of prostatespecific membrane antigen, in patients with metastatic prostate cancer J. Nucl. Med. 53, pp. 1883-1891; 2012.

25. Szabo Z, Mena E, Rowe SP, et al. Initial evaluation of [(18)F]DCFPyL for prostate-specific membrane antigen (PSMA)-targeted PET imaging of prostate cancer Mol. Imaging Biol.17, pp. 565-574; 2015

26. Kelly J, Amora-Coarasa A, Nikolopoulou A, et al. Synthesis and preclinical evaluation of a new class of highaffinity 18F-labeled PSMA ligands for detection of prostate cancer by PET imaging Eur. J. Nucl. Med. Mol. Imaging, 44, pp. 647-661; 2017. 
27. Dietlein M, Kobe C, Kuhnert G, et al. Comparison of [(18)F] DCFPyL and [(68)Ga]Ga-PSMA-HBED-CC for PSMAPET Imaging in patients with relapsed prostate cancer Mol. Imaging Biol. 17, pp. 575-584; 2015.

\section{Cardinale J, Schafer M, Benesova}

M, et al. Preclinical evaluation of $18 \mathrm{~F}-$ PSMA-1007, a new prostate-specific membrane antigen ligand for prostate cancer imaging J. Nucl. Med. 58, pp. 425$431 ; 2017$.

29. Harada N. Kimura H, Onoe S, et al. Synthesis and biologic evaluation of novel 18f-labeled probes targeting prostate-specific membrane antigen for PET of prostate cancer J. Nucl. Med. 57 (2016), pp. 1978-1984.

30. Bouvet M, Wuest HS, Jans M, et al. Automated synthesis of [(18)F]DCFPyL via direct radiofluorination and validation in preclinical prostate cancer models EJNMMI Res, 6, p. 40; 2016.

31. Sasajima T, Ono T, Shimada N, et al. Trans-1-amino-3-18F-

fluorocyclobutanecarboxylic acid (anti18F-FACBC) is a feasible alternative to 11C-methyl-L-methionine and magnetic resonance imaging for monitoring treatment response in gliomas Nucl. Med. Biol. 40, pp. 808-815; 2013.
32. Oka S, Okudaira H, Yoshida Y, et al. Transport mechanisms of trans-1amino-3-fluoro[1-

(14)C]cyclobutanecarboxylic acid in prostate cancer cells Nucl. Med. Biol. 39, pp. 109-119; 2012.

33. Schuster DM, Nanni C, Fanti S. PET tracers beyond FDG in prostate cancer Semin Nucl. Med. 46, pp. 507-521; 2016.

34. Savir-Baruch B, Zanoni L, Schuster DM. Imaging of prostate cancer using fluciclovine PET Clin. 12, pp. 145157; 2017.

\section{Ganapathy $V$, Thangaraju $M$.}

Prasad Nutrient transporters in cancer: Relevance to Warburg hypothesis and beyond Pharmacol Ther. 121, pp. 29-40; 2009.

36. Nakanishi T ad Tamai I. Solute carrier transporters as targets for drug delivery and pharmacological intervention for chemotherapy J. Pharm. Sci, 100 , pp. 3731-3750; 2011.

\section{Oka S, Okudaira H, Ono M, et al.}

Differences in transport mechanisms of trans-1-amino-3-

[18F]fluorocyclobutanecarboxylic acid in inflammation, prostate cancer, and glioma cells: Comparison with L-[methyl$11 \mathrm{C}]$ methionine and 2-deoxy-2-[18F] fluoro-D-glucose Mol. Imaging Biol. 16, pp. 322-329; 2014. 
38. Asano Y, Inoue Y, Ikeda Y, et al. Phase I clinical study of NMK36: A new PET tracer with the synthetic amino acid analogue anti-[18F] FACBC Ann. Nucl. Med. 25, pp. 414-418; 2011.

39. Spratt DE, Gavane S, Tarlinton L, et al. Utility of FDG-PET in clinical neuroendocrine prostate cancer, Prostate, 74, pp. 1153-1159; 2014.

40. Perera M, Papa N, Christidis D, et al. Sensitivity, specificity, and predictors of positive 68Ga-prostate-specific membrane antigen positron emission tomography in advanced prostate cancer: A systematic review and meta-analysis, Eur. Urol. 70 (6), pp. 926-937; 2016.

41. Evangelista L, F Zattoni F, A Guttila A, et al. Choline PET or PET/CT and biochemical relapse of prostate cancer: A systematic review and meta-analysis, Clin. Nucl. Med. 38, pp. 305-314; 2013.

\section{Umbehr MH, Müntener M, Hany}

T, et al. The role of 11C-choline and 18Ffluorocholine positron emission tomography (PET) and PET/CT in prostate cancer: A systematic review and metaanalysis, Eur. Urol. 64, pp. 106-117; 2013.

43. Shen G, Deng H, Hus S. Comparison of choline-PET/CT, MRI, SPECT, and bone scintigraphy in the diagnosis of bone metastases in patients with prostate cancer: A meta-analysis Skeletal Radiol. 43 (11), pp. 1503-1513; 2014.
44. Kitajima K, Murphy RC, Nathan $M A$, et al. Detection of recurrent prostate cancer after radical prostatectomy: Comparison of 11C-choline PET/CT with pelvic multipara metric MR imaging with endorectal, coil. J. Nucl. Med. 55, pp. 223$232 ; 2014$.

45. Ren J, Yuan L, Wen $\boldsymbol{G}$ The value of anti-1-amino-3-18F-fluorocyclobutane1-carboxylic acid PET/CT in the diagnosis of recurrent prostate carcinoma: A metaanalysis, Acta. Radiol, 57. pp. 487-493; 2016.

46. Schuster DM, Nieh PT, Jani AB, et al. Anti-3-[(18) F] FACBC positron emission tomography-computerized tomography and (111) In-capromab pendetide single photon emission computerized tomography-computerized tomography for recurrent prostate carcinoma: Results of a prospective clinical trial. J. Urol. 191, pp. 1446-1453; 2014.

47. Odewole OA, Tade FI, Nieh PT, et al. Recurrent prostate cancer detection with anti-3-[(18)F]FACBC PET/CT: Comparison with CT Eur. J. Nucl. Med. Mol. Imaging, 43, pp. 1773-1783; 2016.

48. Schwenck J, Rempp H, Reischl G, et al. Comparison of 68 Ga-labelled PSMA-11 and 11C-choline in the detection of prostate cancer metastases by PET/CT, Eur. J. Nucl. Med. Mol. Imaging, 44, pp. 92-101; 2017. 